\title{
Respuesta a everolimus en un neonato con rabdomioma cardiaco asociado con el complejo esclerosis tuberosa. Reporte de caso
}

\author{
Everolimus response in a newborn with cardiac \\ rhabdomyoma associated to tuberous sclerosis complex: \\ Case report
}

María del Carmen Esmer-Sánchez, ${ }^{1}$ Martha Elizabeth Rubio-Hernández, ${ }^{2}$ Juan José Morales-Ibarra, ${ }^{3}$ Jorge Guillermo Reyes-Vaca, ${ }^{4}$ Dairy Marlenne Margarita Villegas-Valdez, ${ }^{4}$ Claudia Lizbeth Gómez-Elías, ${ }^{3}$ Antonio Bravo-Oro ${ }^{3}$

\begin{abstract}
Resumen
ANTECEDENTES: El rabdomioma cardiaco tiene una incidencia posnatal de 1 en 40,000 y comprende $45-75 \%$ de los tumores detectados en la etapa prenatal, con 70 a $80 \%$ asociados con el complejo de esclerosis tuberosa.

CASO CLíNICO: Paciente pediátrico con un tumor en la pared del ventrículo izquierdo; se detectó a las 18 semanas de gestación. Al nacimiento tuvo taquicardia y dificultad respiratoria, por lo que comenzó a tratársele con digoxina y furosemida. Se sospechó un rabdomioma cardiaco porque la resonancia mostró múltiples túberes corticales, displasia marginal lineal parietal izquierda y nódulos subependimarios; el complejo de esclerosis tuberosa se confirmó por clínica, sin análisis genético confirmatorio. Comenzó a tratarse con everolimus y, un mes después, se advirtió la reducción del tamaño del tumor y la mejoría en la capacidad de contracción del ventrículo izquierdo. A los cinco meses el paciente se encontraba libre de signos y síntomas de insuficiencia cardiaca; a los 10 meses cursó con episodios de epilepsia y a los 24 meses la ecocardiografía y el neurodesarrollo se reportaron normales; sin embargo, continuó con antiepilépticos y everolimus, sin complicaciones.
\end{abstract}

CONCLUSIONES: El inmunosupresor everolimus es una alternativa de tratamiento para pacientes con rabdomiomas cardiacos y esclerosis tuberosa, con tumores de gran tamaño o localizados en sitios de difícil acceso que complican la decisión quirúrgica. PALABRAS CLAVE: Rabdomioma cardiaco; complejo de esclerosis tuberosa; ventrículo izquierdo; insuficiencia respiratoria; prueba genética; insuficiencia cardiaca; Everolimus

\section{Abstract}

BACKGROUND: Cardiac rhabdomyoma has a postnatal incidence of 1 in 40,000 and comprises $45-75 \%$ of tumors that are detected at the prenatal stage, with 70 to $80 \%$ of them associated with the tuberous sclerosis complex.

CLINICAL CASE: Male fetus with a mass located over the wall of the left ventricle identified at 18 weeks of gestation, after birth developed tachycardia and respiratory distress; treatment with digoxin and furosemide was initiated. Cardiac rhabdomyoma was suspected; brain magnetic resonance showed multiple cortical tubers, left parietal linear marginal dysplasia and subependymal nodules, tuberous sclerosis complex diagnosis was made without confirmatory genetic testing. Everolimus was started; one-month later reduction of the tumor was observed with clinical improvement. After five-month the mass shrink and heart failure disappeared, and furosemide and digoxin were stopped. At 10 months he started with epilepsy, at 2 years the echocardiography and the neurodevelopment was normal, he continues with antiepileptic drugs and Everolimus without side effects.

CONCLUSIONS: Everolimus is an alternative for the treatment of cardiac rhabdomyomas in patients with tuberous sclerosis with large tumors or surgically unreachable locations. KEYWORDS: Cardiac rhabdomyoma; Tuberous sclerosis complex; Left ventricle; Respiratory distress; Genetic testing; Heart failure; Everolimus.

\footnotetext{
${ }^{1}$ Departamento de Genética.

2 Departamento de Cardiología Pe-

diátrica.

${ }^{3}$ Departamento de Neurología Pediátrica.

${ }^{4}$ Departamento de Radiología.
}

Hospital Central Dr. Ignacio Morones Prieto.

Recibido: 20 de enero 2020

Aceptado: 2 de septiembre 2020

Correspondencia

Antonio Bravo Oro

neurologobravo@gmail.com

Este artículo debe citarse como Esmer-Sánchez MC, Rubio-Hernández ME, Morales-Ibarra JJ, Reyes-Vaca JG, Villegas-Valdez DMM, Gómez-Elías CL, Bravo-Oro A. Respuesta a everolimus en un neonato con rabdomioma cardiaco asociado con el complejo esclerosis tuberosa. Reporte de caso. Acta Pediatr Méx 2020; 41(5): 208-14. 


\section{ANTECEDENTES}

El rabdomioma cardiaco es el tumor benigno más frecuente en niños, su incidencia posnatal es de 1 caso por cada 40,000 nacimientos. En la mayoría de los casos su curso es benigno, con regresión espontánea entre los 2 y 4 años de vida. El rabdomioma cardiaco comprende 45 a $75 \%$ de los tumores que se detectan en la etapa prenatal y 70 a $80 \%$ de ellos se asocian con el complejo de esclerosis tuberosa. ${ }^{1,2}$

El complejo de esclerosis tuberosa es un padecimiento autosómico dominante con mutaciones en TSC1 (9q34) y TSC2 (16p13); ambos genes codifican proteínas que estimulan la vía de proliferación y crecimiento celular mTOR (mamalian target of rapamicyn). Sus criterios diagnósticos se actualizaron en 2012 (Cuadro 1). El rabdomioma cardiaco, al igual que los nódulos subependimarios, hamartomas corticales y astrocitoma subependimario de células gigantes pueden aparecer en la etapa prenatal. ${ }^{3}$ En 2010, la FDA aprobó al everolimus para el tratamiento de pacientes con astrocitoma subependimario de células gigantes inoperable y el angiomiolipoma renal, asociados con el complejo de esclerosis tuberosa. ${ }^{4}$ Desde entonces, al menos 25 casos de rabdomioma cardiaco congénito, tratados con everolimus, se han descrito con adecuada reducción del tamaño tumoral. . $^{5-9}$

\section{CASO CLÍNICO}

Paciente pediátrico, nacido del segundo embarazo, sin antecedentes familiares relevantes. En el ultrasonido de las 18 semanas de gestación se identificó un tumor en la pared del ventrículo izquierdo de $3.0 \times 2.5 \mathrm{~cm}$. El niño nació a las 37.3 semanas, por vía cesárea, con polipnea, taquicardia y ligera hepatomegalia, cardiomegalia, congestión pulmonar. El ecocardiograma mostró: ritmo sinusal, masa sólida que deformaba la pared anterior del ventrículo izquierdo, de $3.0 \mathrm{x}$ $2.2 \mathrm{~cm}$, que afectaba la contractilidad y obstruía el flujo. Figura 1a

El tratamiento se inició con digoxina, furosemida y levocarnitina. La resonancia magnética de cráneo con nódulos subependimarios y hamartomas subcorticales (Figura $\mathbf{2}$ a-b), la evaluación oftalmológica, cutánea y el ultrasonido renal se reportaron dentro de la normalidad. Se esta-

Cuadro 1. Criterios para complejo de esclerosis tuberosa

\section{A. Criterios diagnósticos genéticos}

- La identificación de una mutación patogénica de TSC1 o TSC2 en el ADN obtenido de tejido normal es diagnóstico definitivo.

- Otras variantes cuyo efecto en la función es incierta no cumplen los criterios y no son suficientes para establecer el diagnóstico.

- Entre 10 y $25 \%$ de los pacientes no tienen mutación identificada por estudios genéticos convencionales y un estudio normal no excluye el diagnóstico o no tiene ningún efecto en la utilización de los criterios diagnósticos clínicos para complejo de esclerosis tuberosa.

\section{B. Criterios diagnóstico-clínicos}

Criterios mayores

- Mancha hipomelanótica (3 o más de al menos 5 mm de diámetro).

- Angiofibroma (3 o más) o placa cefálica fibrosa.

- Fibroma ungueal (2 o más).

- Parche Shagreen.

- Hamartomas retinianos múltiples

- Displasia cortical (túberes o líneas de migración radial de la sustancia blanca cerebral).

- Nódulos subependimarios

- Astrocitoma subependimario de células gigantes

- Rabdomioma cardiaco

- Linfangioleiomiomatosis

- Angiomiolipomas renales (2 o más)

Nota: la combinación de estos dos últimos criterios ameritó otro criterio mayor más para definir el diagnóstico.

\section{Criterios menores}

Lesiones en piel de "confeti"

- Lesiones del esmalte dental (3 o más).

- Fibromas intraorales (2 o más)

- Parche acrómico de la retina.

- Quistes renales múltiples.

- Hamartomas no renales.

*Tomado de Northrup H, et al. ${ }^{3}$ 
bleció el diagnóstico definitivo de complejo de esclerosis tuberosa.

Se obtuvo el consentimiento para indicar everolimus y divulgar, con fines académicos, los desenlaces. Los especialistas del departamento de cirugía cardiovascular consideraron que el rabdomioma cardiaco no reunía los requisitos para el tratamiento quirúrgico, por la localización del tumor en la pared ventricular; al cuarto día de vida se inició la administración de everolimus, por vía oral, a dosis de $4.5 \mathrm{mg} / \mathrm{m}^{2} \mathrm{SC} /$ día. Las con-
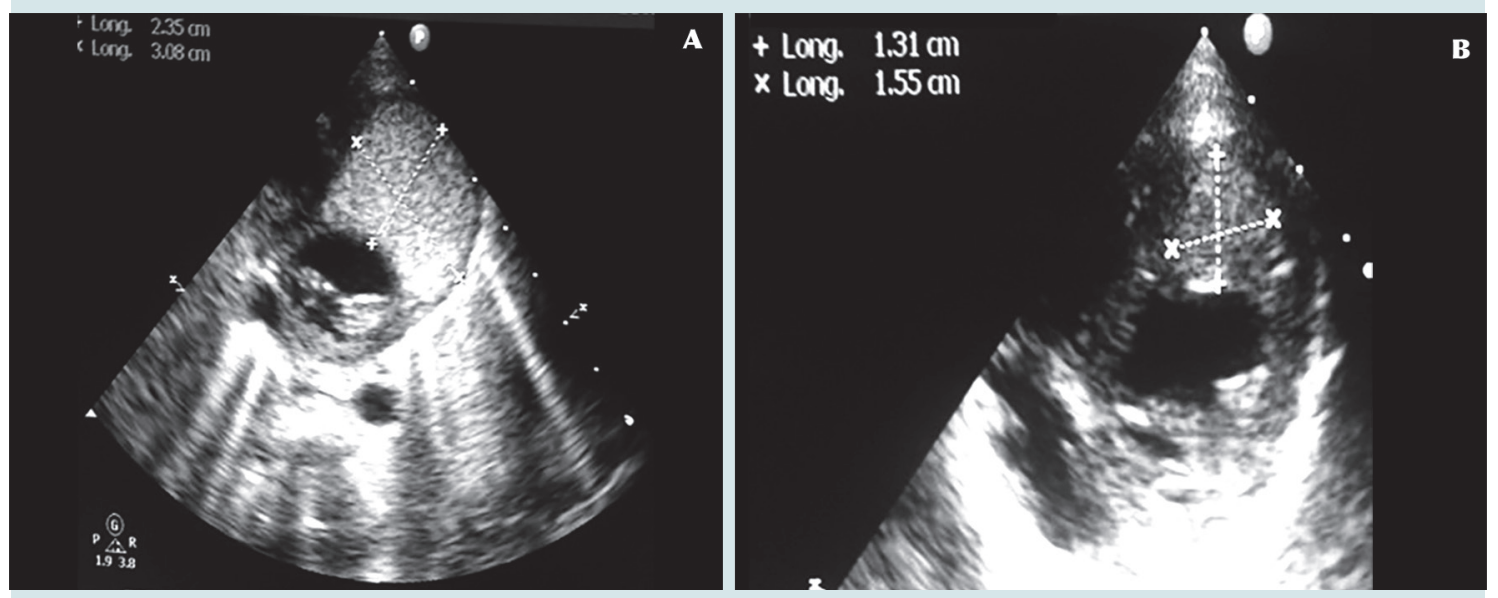

Figura 1. A) Primera ecocardiografía a las 12 horas de vida que muestra una lesión sólida en el ventrículo izquierdo sugerente de rabdomioma. B) Segunda ecocardiografía al mes de vida, con disminución importante del tamaño de la lesión del ventrículo izquierdo.
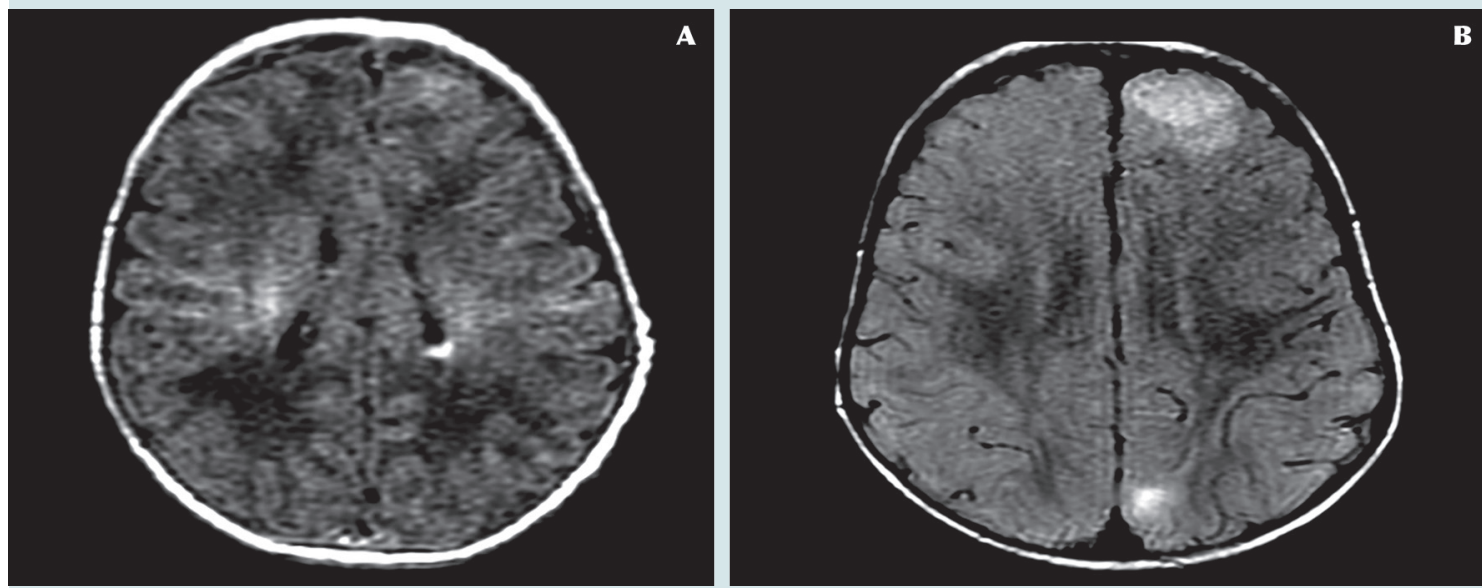

Figura 2. A) Imagen obtenida a los 5 días de vida. Corte axial potenciado en T1, en el que se observan nódulos subependimarios hiperintensos y hamartomas o túber subcorticales hipointensos. B) A los 14 meses de vida. Corte axial potenciado en T1 se observa aumento de tamaño y de la cantidad de hamartomas subcorticales. 
centraciones séricas de everolimus se encontraron en $26.8 \mathrm{ng} / \mathrm{mL}(5-10 \mathrm{ng} / \mathrm{mL})$; a las dos semanas se ajustó la dosis. Al mes, se encontraron en 5.7 $\mathrm{mg} / \mathrm{mL}$ y el rabdomioma cardiaco midió $1.3 \mathrm{x}$ $1.5 \mathrm{~cm}$, con mejoramiento de la contractilidad del ventrículo izquierdo. Figura 1b

A los cinco meses, la masa midió $0.8 \times 0.8 \mathrm{~cm}$, FEV $176 \%$. A los 13 meses de vida $0.8 \times 0.7 \mathrm{~cm}$ y la FEV1 de $70 \%$ y asintomático; se suspendieron la furosemida y la digoxina. A los 3 meses de edad el paciente tuvo elevación de triglicéridos $(312 \mathrm{mg} / \mathrm{dL})$, que se normalizaron a los 6 meses (132 mg/dL). A los 10 meses comenzó con epilepsia focal, que controló con valproato de magnesio, topiramato y vigabatrina. La resonancia magnética de cráneo, a los 14 meses de vida, mostró aumento de tamaño de los nódulos subependimarios y de los hamartomas subcorticales. A los 2 años se documentó la desaparición del tumor en el ecocardiograma, con neurodesarroIlo normal; continuó con antiepilépticos y con concentraciones de everolimus en $8.5 \mathrm{ng} / \mathrm{mL}$.

\section{DISCUSIÓN}

Los rabdomiomas cardiacos se manifiestan en múltiples masas redondas, homogéneas, debidamente delimitadas, hiperecogénicas, asimétricas. Solo $10 \%$ son una lesión única, como en el paciente del caso. Su localización en el ventrículo izquierdo ocurre en 93\%. Su tamaño es variable y van desde milímetros hasta pocos centímetros. Uno de los más grandes, diagnosticado por ecocardiograma fetal, midió $60 \times 45$ × 60 mm. Las series de casos grandes han concluido que la mayor morbilidad y mortalidad ocurre cuando miden más de $30 \mathrm{~mm}^{2,10-12}$

Con frecuencia, el diagnóstico es un hallazgo en el ultrasonido prenatal; $13.7 \%$ se detectan antes de la semana 24 de gestación. Posterior al diag- nóstico inicial deben practicarse ultrasonidos periódicos para identificar los signos de insuficiencia cardíaca congestiva o las alteraciones en el ritmo. Los rabdomiomas cardiacos aumentan en volumen entre el segundo y el tercer trimestre, hasta el período neonatal, quizá favorecido por los estrógenos de la madre.

Con el tiempo, las células de rabdomioma cardiaco pierden la capacidad de dividirse. ${ }^{6}$ Jozwiak y su grupo describieron la desaparición parcial en $50 \%$ de los casos y la regresión completa en $18 \%$ y en $4 \%$ crecen o aparecen de novo. ${ }^{2}$

Los rabdomiomas cardiacos son asintomáticos en $21.2 \%$ de los casos; en $33.3 \%$ se encuentra un soplo cardíaco, arritmias en $24.2 \%$ o cianosis en $6.1 \%$, que disminuyen gradualmente. Pocos casos resultan con obstrucción del flujo ventricular, insuficiencia valvular, arritmias auriculares y ventriculares, síndrome de Wolff-Parkison-White, insuficiencia cardiaca congestiva, choque cardiaco o hidrops fetal, con mortalidad menor a $10 \%$ relacionada con el tamaño y localización. ${ }^{13}$

El pronóstico es mejor si no hay descompensación hemodinámica in útero o en los primeros seis meses de vida. La conducta médica suele ser expectante y conservadora porque la mayoría involucionan espontáneamente, sin necesidad de tratamiento. Solo se considera la intervención médica o quirúrgica ante el riesgo de muerte súbita en pacientes con obstrucción al flujo de entrada y salida, inestabilidad hemodinámica, disfunción valvular o arritmias.

Las indicaciones de la cirugía incluyen: síntomas significativos en combinación con una masa extraíble. ${ }^{14,15}$

La rapamicina fue el primer inhibidor de la vía mTOR, con potente capacidad antiproliferativa e 
inmunosupresora. El everolimus es un derivado de la rapamicina con mayor estabilidad, solubilidad y biodisponibilidad. ${ }^{16}$ En la bibliografía está documentada la indicación de everolimus en casos de inestabilidad hemodinámica y rabdomiomas cardiacos inoperables., ${ }^{4,5}$, En los cinco casos descritos por Dhulipudi y su grupo la dificultad respiratoria fue la manifestación más común; todos los pacientes mostraron regresión de los síntomas hemodinámicos y del tamaño del rabdomioma cardiaco. Un caso tuvo infección por varicela 6 semanas después del comienzo del everolimus; otro caso con múltiples rabdomiomas cardiacos grandes y obstrucción de la cavidad ventricular derecha tuvo muerte cardiaca súbita. ${ }^{9}$

La velocidad de disminución del tamaño tumoral es 11.8 veces más rápida con everolimus comparada con la evolución natural del rabdomioma cardiaco. ${ }^{17} \mathrm{El}$ caso del paciente fue de regresión del tumor a partir del día 14 del tratamiento con everolimus a dosis de $4.5 \mathrm{mg} / \mathrm{m}^{2} \mathrm{SC} /$ día, conforme al esquema recomendado para astrocitoma subependimario de células gigantes, aunque a los 15 días se redujo la dosis a días alternos por alcanzar concentraciones de toxicidad.

Otros autores han indicado, eficazmente, la dosis de 0.25 mg cada 12 horas, dos veces a la semana, señalando la importancia de contar con recomendaciones para recién nacidos. ${ }^{5}$ El everolimus oral se absorbe rápidamente y alcanza la concentración máxima después de 1.3-1.8 h, su vida media biológica es de 30 h, las concentraciones se estabilizan en 7 días. Se metaboliza, sobre todo, por CYP3A4 y sus efectos adversos más comunes son: estomatitis, eritema, infecciones de las vías respiratoria y gastrointestinal, elevación del colesterol, triglicéridos y enzimas hepáticas. ${ }^{4}$ En 24 meses de indicar everolimus se documentó una discreta elevación de los triglicéridos que no requirió tratamiento farmacológico; este hallazgo fue previamente informado por otros autores.

Los hallazgos clínicos en pacientes con complejo de esclerosis tuberosa, que aparecen más temprano son: rabdomioma cardiaco y astrocitoma subependimario de células gigantes. En el caso aquí comunicado, a partir del nacimiento se confirmaron los criterios diagnósticos suficientes para complejo de esclerosis tuberosa. Los pacientes con rabdomioma cardiaco neonatal, asociados con el complejo de esclerosis tuberosa, tienen convulsiones en $80.6 \%$ de los casos, posteriormente pueden sufrir discapacidad intelectual, retraso psicomotor, trastornos del comportamiento y del lenguaje. La mayoría muestran túberes corticales, nódulos subependimarios, displasia cortical y astrocitoma subependimario de células gigantes; es frecuente que en un mismo paciente coexistan dos o más lesiones. Todos los casos resultan con máculas hipomelanóticas, angiofibroma facial en $45.1 \%$, placas de shagreen en $19.3 \%$, hamartoma retiniano en $41.9 \%$, angiomiolipoma renal en $16.1 \%$ y quistes renales en $6.4 \%{ }^{18}$

El tratamiento con everolimus consigue disminuir la cantidad de las crisis, con mejores resultados en pacientes con concentraciones séricas entre 9-15 vs 3-7 ng/mL. ${ }^{19}$ En el paciente del caso las crisis convulsivas se iniciaron a los 10 meses de vida, asociadas con fiebre y persistencia aun recibiendo tres antiepilépticos y concentraciones séricas en $8.7 \mathrm{ng} / \mathrm{mL}$. Los pacientes que inician con epilepsia antes de los 12 meses tienen mayor riesgo de trastornos en el neurodesarrollo, incluido el autismo; este caso lleva un neurodesarrollo motor y cognitivo dentro de lo esperado para la edad. ${ }^{18}$ Los túberes corticales se incrementaron en cantidad. En el estudio de resonancia magnética de control del 
paciente, no hubo reportes que documentaran la evolución de estas lesiones en el sistema nervioso central, con el consumo crónico del inhibidor mTor porque en otros órganos: riñón, pulmón o retina sí es eficaz para disminuir el volumen de los tumores. ${ }^{19}$

La utilidad clínica de contar con un estudio de ADN confirmatorio es limitada en cuanto a la gravedad del padecimiento, aunque se conoce que TSC2 produce un fenotipo más grave que TSC 1. Los individuos con variantes patogénicas en TSC2 están en mayor riesgo de neoplasia renal, discapacidad intelectual, trastorno del espectro autista y espasmos infantiles. Las mujeres con variantes patogénicas en TSC2 tienen mayor frecuencia de linfangioleiomiomatosis. El rabdomioma cardiaco y la enfermedad poliquística renal tienden a ser más frecuentes en los casos con variantes patogénicas en TSC2.2,3 El padecimiento no afecta la fertilidad, por lo que la mayor utilidad de contar con el análisis de mutaciones es el asesoramiento genético preciso y dar fundamento para estudios prenatales o preimplantación que permitan prevenir la ocurrencia del complejo de esclerosis tuberosa en la descendencia del afectado.

\section{CONCLUSIONES}

El pronóstico de los pacientes con rabdomiomas cardiacos se determina, principalmente, por el tamaño y la ubicación de la lesión. No existen lineamientos nacionales o internacionales para indicación del everolimus en rabdomioma cardiaco. La evidencia en la bibliografía permite considerarlo una herramienta terapéutica útil cuando el tumor afecta el estado hemodinámico. La prescripción de everolimus debe vigilarse estrechamente con evaluaciones que permitan ajustar las dosis para evitar los eventos adversos.

\section{REFERENCIAS}

1. Kwiatkowska J, et al. Cardiac tumors in children: A 20year review of clinical presentation, diagnostics, and treatment. Adv Clin Exp Med. 2017; 26 (2): 319-26. doi. 10.17219/acem/6212.

2. Jozwiak S, et al. Clinical and genotype studies of cardiac tumors in 154 patients with tuberous sclerosis complex. Pediatrics. 2006; 118 (4): e1146-51. https://doi. org/10.1542/peds.2006-0504.

3. Northrup $\mathrm{H}$, et al. Tuberous sclerosis complex diagnostic criteria update: recommendations of the 2012 International Tuberous Sclerosis Complex Consensus Conference. Pediatr Neurol. 2013; 49 (4): 243-54. doi. 10.1016/j. pediatrneurol.2013.08.001.

4. Davies M, et al. Management of Everolimus-associated adverse events in patients with tuberous sclerosis complex: A practical guide. Orphanet J Rare Dis. 2017; 12 (1): 35. doi.10.1186/s13023-017-0581-9.

5. Demir HA, et al. Everolimus: a challenging drug in the treatment of multifocal inoperable cardiac rhabdomyoma. Pediatrics. 2012; 130 (1): e243-7. doi. 10.1542/ peds.2011-3476.

6. Miczoch E, et al. Prenatal diagnosis of giant cardiac rhabdomyoma in tuberous sclerosis complex: a new therapeutic option with Everolimus. Ultrasound Obstet Gynecol. 2015; 45 (1): 618-21. doi. 10.1002/uog.13434.

7. Colaneri M, et al. Everolimus-induced near-resolution of giant cardiac rhabdomyomas and large renal angiomyolipoma in a newborn with tuberous sclerosis complex. Cardiol Young. 2016; 26 (8): 1025-8. doi. 10.1017/ S1047951116000421.

8. Jeng-Sheng $\mathrm{Ch}$, et al. Regression of neonatal cardiac rhabdomyoma in two months through low-dose Everolimus therapy: a report of three cases. Pediatr Cardiol. 2017; 38 (7): 1478-84. doi.10.1007/s00246-017-1688-4.

9. Dhulipudi B, et al. Symptomatic improvement using Everolimus in infants with cardiac rhabdomyoma. Ann Pediatr Cardiol. 2019; 12 (1): 45-8. doi. 10.4103/apc.APC_79_18.

10. Ide $\mathrm{T}$, et al. Prediction of postnatal arrhythmia in fetuses with cardiac rhabdomyoma. J Matern Fetal Neonatal Med. 2019; 32 (15): 2463-8. doi:10.1080/14767058.20 18.1438402.

11. Sciaca $\mathrm{P}$, et al. Rhabdomyomas and tuberous sclerosis complex: our experience in 33 cases. BMC Cardiovasc Disord. 2014; 14: 106. doi:10.1186/1471-2261-14-66.

12. Park $\mathrm{H}$, et al. Sirolimus therapy for fetal cardiac rhabdomyoma in a pregnant woman with tuberous sclerosis. Obstet Gynecol Sci. 2019; 62 (4): 280-4. doi:10.5468/ ogs.2019.62.4.280.

13. Öztunç F, et al. Everolimus treatment of a newborn with rhabdomyoma causing severe arrhythmia. Cardiol Young. 2015; 25 (7): 1411-4. doi:10.1017/S1047951114002261. 
14. Choudhry S, et al. Rapid resolution of cardiac rhabdomyomas following Everolimus therapy. BMJ Case Rep. 2015: 9; 2015. pii: bcr2015212946. doi: 10.1136/bcr-2015-212946.

15. Wagner $R$, et al. Oral Everolimus for treatment of a giant left ventricular rhabdomyoma in a neonate-rapid tumor regression documented by real time 3d echocardiography. Echocardiography. 2015; 32 (12): 1876-9. doi:10.1111/echo.13015.

16. Franz DN, et al. Efficacy and safety of Everolimus for subependymal giant cell astrocytomas associated with tuberous sclerosis complex (EXIST-1): a multicentre, randomised, placebo-controlled phase 3 trial. Lancet. 2013; 381 (12): 125-32. doi:10.1016/S0140-6736(12)61134-9.
17. Aw F, et al. Accelerated Cardiac Rhabdomyoma Regression with Everolimus in Infants with Tuberous Sclerosis Complex. Pediatr Cardiol. 2016; 38 (2): 394-400.

18. Orlova KA, Crino PB. The tuberous sclerosis complex. Ann N Y Acad Sci. 2010; 1184: 87-105. doi: 10.1111/j.17496632.2009.05117.x

19. French JA, et al. Adjunctive Everolimus therapy for treatment-resistant focal-onset seizures associated with tuberous sclerosis (EXIST-3): a phase 3, randomised, double-blind, placebo-controlled study. Lancet. 2016; 388 (10056): 2153-63. doi. 10.1016/S01406736(16)31419-2. 